\title{
Social Dynamics of Ebola Virus Disease: A Case of Bundibugyo District, Uganda
}

\author{
Clovice Kankya1,2*\#, Daisy Nabadda1,2\#, Consolata Kabonesa ${ }^{2}$, Luke Nyakarahuka1, \\ James Muleme', Samuel Okware ${ }^{3}$, Richard Asaba ${ }^{2}$
}

\author{
${ }^{1}$ Department of Biosecurity, Ecosystems and Veterinary Public Health, College of Veterinary Medicine, \\ Animal Resources and Biosecurity (COVAB), Makerere University, Kampala, Uganda \\ ${ }^{2}$ Department of Gender, School of Women and Gender Studies, College of Humanities and Social Sciences (CHUSS), \\ Makerere University, Kampala, Uganda \\ ${ }^{3}$ Uganda National Health Research Organization, Entebbe, Uganda \\ Email: ‘clokankya@yahoo.com, clokankya@gmail.com,ndjuuko@gmail.com,ndjuuko@yahoo.com,ckabonesa@ss.mak.ac.ug, \\ consoloata.kabonesa@gmail.com, mulemej@gmail.com,okwares@gmail.com,rasabab@yahoo.com
}

How to cite this paper: Kankya, C., Nabadda, D., Kabonesa, C., Nyakarahuka, L., Muleme, J., Okware, S. and Asaba, R. (2019) Social Dynamics of Ebola Virus Disease: A Case of Bundibugyo District, Uganda. Health, 11, 108-128.

https://doi.org/10.4236/health.2019.111011

Received: December 23, 2018

Accepted: January 27, 2019

Published: January 30, 2019

Copyright $\odot 2019$ by author(s) and Scientific Research Publishing Inc. This work is licensed under the Creative Commons Attribution International License (CC BY 4.0).

http://creativecommons.org/licenses/by/4.0/

\begin{abstract}
Background: Ebola Virus Disease (EVD) presents with a high global mortality and is known to be a highly infectious disease with devastating and gendered effects on the social fabric, yet most of the science has focused on the disease's biology. However, little has been documented with regard to the gender and social aspects of Ebola Virus Disease (EVD) in two sub counties (Kikyo and Bundibugyo Town Council) in Bundibugyo District in Western Uganda. The study was set to examine the gender differences in the level of knowledge, attitudes and perceptions about EVD. Methods: The study employed a cross-sectional design using both quantitative and qualitative data collection methods. A structured questionnaire was administered to 254 respondents, $50 \%$ of whom were women. Simple random sampling was used to select the participants. Questionnaire data were analysed using SPSS at univariate and bivariate levels. Qualitative methods such as key informant interviews (with 6 participants) and Focus Group Discussions (three, one with men alone, another with women alone and the last one with both men and women) were also used to collect additional information from participants. Results: The findings indicated that socio-demographically, the majority (35\%) of the respondents were aged between 20 and 29 years, $53 \%$ of whom were females. More women (about 56\%) compared to men (44\%) attained secondary education while more men (about $51 \%$ versus $49 \%$ of the women) reported that they were married. In terms of religion, the majority of the survey participants were Catholics (59\% females and $49 \%$ males). With regard to communities' knowledge about EVD, there was no significant relationship between men and women in terms of prior knowledge about EVD, risk
\end{abstract}


factors and control measures. However, slightly more males (about $51 \%$ ) than females (49\%) had heard about EVD and more males (about 52\%) than females (48\%) admitted that they were at risk of contracting the disease. On the control measures, slightly more females than males (about $53 \%$ vs. $47 \%$ respectively) proposed avoiding contact with infected persons. The results further revealed that more males (about 51\%) than females (49\%) were willing to relate with EVD survivors, and this was due to the latter's fear of contracting the disease. The major devastating effects of EVD that were reported included loss of lives, disruption of peoples movements, isolation of people, disruption of children's school activities, stigma and discrimination of survivors. More females (about 52\%) than males (about 48\%) reported that EVD survivors were discriminated during the EVD outbreak in Bundibugyo District. Conclusions: This study has shown that whereas both men and women demonstrate average knowledge about EVD, there is need for more training and sensitization targeting women who have delusions about the severity of the disease, its risk factors, stigma and the integration of survivors in the affected communities. Communities also need to be sensitized about the gender roles that increase both the burden of EVD and the risk of men and women contracting the disease. This will lead to more culturally sensitive responses to EVD outbreaks in future.

\section{Keywords}

Ebola Viral Disease (EVD), Gender, Bundibugyo District, Uganda

\section{Introduction}

Ebola Virus Disease (EVD) is one of the highly infectious emerging Viral Haemorrhagic Fevers (VHFs) caused by infection with one or more of the Ebola virus species. It was first discovered in 1976 near the Ebola River in what is now the Democratic Republic of the Congo. A second outbreak occurred shortly afterwards in Nzara Town South Sudan in the Western Equatorial State. Since then, major and minor outbreaks have occurred sporadically in Africa [1]. There is no known treatment for EVD and case fatality rate is terribly high. Several other deadly VHFs exist and these include: Marburg, Lassa fever, Crimean-Congo haemorrhagic fever, and Rift Valley fever, among others. Ebola can cause disease in humans and nonhuman primates (monkeys, gorillas, and chimpanzees). Ebola is caused by an infection with a virus of the Filoviridae family and the Ebolavirus genus. Currently, there are five identified Ebola virus species, four of which have caused disease in humans and these include: the Ebola virus (Zaire ebolavirus), Sudan virus (Sudan ebolavirus), Taï Forest virus (Taï Forest ebolavirus, formerly Côte d'Ivoire Ebola virus), and newly discovered [2] Bundibugyo virus (Bundibugyoebolavirus). The fifth species is the Reston virus (Reston ebolavirus) which has had one mild case of a Zoologist in Ivory Coast but no outbreak in nonhuman primates [3]. The Zaire and the Sudan subtypes are the most severe 
with case fatality rate between $60 \%$ and $90 \%$ on average. The Bundibugyo outbreak is less lethal with a lower case fatality rate of $34 \%$. The time period between contracting the disease and showing symptoms after exposure ranges from 2 to 21 days [4]. The natural reservoir host of Ebola viruses still remains unknown.

Ebola is described by [5] as a "severe, acute viral illness", with initial symptoms including a sudden onset of fever, intense weakness, muscle pain, fatigue, headache and a sore throat, followed by vomiting, diarrhoea, rash, impaired kidney and liver function, abdominal pain and in some cases, both internal and external bleeding [6]. There is currently no cure for Ebola and the disease seriously affects several organs in the body leading to multiple organ failure, shock and death within days. Vital organs like the liver, kidney and the spleen are severely and irreversibly damaged. EVD outbreaks principally occur in Central and Equatorial Africa with major outbreaks in the Democratic Republic of Congo (DRC), Gabon, Congo Brazzaville, Uganda, Gabon and South Sudan. The worst recent outbreak occurred in West Africa between 2014 and 2015 during which nearly 28,000 cases and nearly 11,000 deaths were reported in Sierra Leone, Liberia and Guinea. Sporadic cases have been reported in USA, Philippine, Italy (Reston) and some imported cases also reported in Europe. In 2015, many African countries in West and central Africa were severely affected including Liberia, Sierra Leone, Guinea, and Nigeria [7]. A total of 28,256 suspected cases and 11,306 deaths in West Africa alone were recorded by the end of the outbreak in September 2015 [8].

On the basis of evidence and the nature of similar viruses, researchers believe that the virus is animal-borne and that bats are the most likely reservoir [9]. In Uganda for example, a young girl who suddenly died after 3 hours of showing the signs of EVD in Luwero District was believed to have acquired the virus around her home since she had not left the home for the past 3 months [10]. Direct contact with body fluids of Ebola patients is a major mode of transmission. It is also believed that the virus enters the population through contact with body fluids of non-human primates such as bats and chimpanzees through hunting of game for food. It is also believed that bats may be the major reservoir carrying the infection as $5 \%$ of bats without symptoms were found to be positive [2]. The ebola virus has also been isolated in semen up to 61 days post recovery, thereby increasing the potential through for sexual transmission. Ebola survivors often suffer from ongoing frustrations like joint and muscle pains, chest pain and vision problems [3].

Despite its significance as a highly infectious disease, most of the science has focused on the biology of EVD. The public health, management and care aspects of Ebola have received relatively little attention. Yet Ebola is closely associated and fuelled by poor socio economic weakness in society. In Africa gender differences emerge as women often are care providers for relatives in low income settings and in very poor sanitary conditions. On the other hand men often hunt 
game for food thereby exposing themselves and interfacing very closely with wildlife.

The sociological and gender aspects of the disease such as community knowledge, experience and impacts are only beginning to gain attention in Africa [11]. In Gulu, Uganda the majority (64\%) of the 424 cases were women [11]. In Sierra Leone for example, it has been reported that women are more affected by EVD than men. The sex differential incidence status in this case was of $56.7 \%$ for females and $43.3 \%$ for males respectively [12]. Available evidence from West Africa and Gulu Uganda further shows that Ebola disproportionately affects women due to their care giving role that increases transmission and resultant death. Women also bear the social burden of the disease as they play a leading role in caring for orphans and school-going children. In addition, most of the healthcare providers such as nurses are often women [13] [14]. This disproportionate sex differential scenario relates to the social risk factors that women face which increase their vulnerability to EVD, largely due to their care giving roles at household and community levels. The differentiated impact is traced to socially prescribed gender norms, behaviour and the gendered division of labour between men and women that perpetuate gender inequality [12]. In Uganda, there is limited evidence of sociological and anthropological studies on EVD. Thus, this study was undertaken in order to understand communities' response to EVD and its associated gender and social aspects in one of the areas where an outbreak has previously occurred in 2007 and that is Bundibugyo District in western Uganda.

Uganda has documented a total of six Ebola outbreaks since the year 2000, including the outbreak in Bundibugyo District in 2007 [11] [15]. The Ugandan government through the Ministry of Health mounted a national response, which contained these outbreaks with varying degrees of success. Most of the time EVD outbreaks were managed by the National Ebola Task Force and district rapid response committees. They were supported international agencies led by the WHO. The activities of the various agencies and partners were integrated into a jointly agreed national strategy and plan [11]. The major response was active case search and surveillance, public education and social mobilisation, isolation and care. The care of post Ebola survivors and orphans was an additional desirable activity. A laboratory centre was established at the Uganda Virus Research Institute (UVRI) at Entebbe [16] for early detection and diagnosis. Early detection and action provided the best outcome in the Luwero outbreak of 2011 [10]. This outbreak was limited to a single case fatality.

Despite the above initiatives the threat of EVD still remains due to inadequate health systems for early detection and surveillance. Focusing on care and prevention of EVD is important but it is not enough. Understanding community perceptions of EVD, its causes and people's knowledge, attitudes, beliefs and myths and the gender issues there-in are key in preventing the reoccurrence of the disease and management of the disease in Uganda and worldwide. Ethnographic parameters for the local population too are critically important in order 
that local cultural practices, misperceptions and beliefs which posed challenges to disease control and need to be addressed because they normally hinder efforts to stop the spread of EVD [13]. In addition, understanding men and women's knowledge and perceptions about EVD is key aspect. The practices, myths and beliefs could be another key in influencing health related behaviours and the success of any public health interventions because Ebola outbreaks cannot be predicted [17].

Thus, understanding community's knowledge and perceptions of the disease, fear, stigma, beliefs and practices of EVD from a gender perspective improves prevention measures and reduces risks of the disease. Transmission and prevention of Ebola is dependent on complex factors including the socio-cultural, people's knowledge, attitudes and individual perception of the disease. To date, few studies have documented the gender and ethnographic differences in the affected communities. Few studies on knowledge, attitudes, perception, beliefs, and practices about EVD are available locally.

\section{Materials and Methods}

\subsection{Study Design}

The study took on a cross-sectional design. Both qualitative and quantitative data collection methods were used to gather data from July to November 2015. The cross-sectional research design was adopted in order to elicit information on the situation from a sample of the population at one point in time and to document EVD experiences from various groups in Bundibugyo District. This design was also adopted because it enables the examination of functional relationships in this case through describing beliefs, practices, experiences and effects of Ebola Virus Disease from a gender perspective. The quantitative design was vital in eliciting the generalised gendered knowledge, attitudes, perceptions, beliefs, management, treatment and effects of EVD in Bundibugyo District. The qualitative approaches allowed the collection of data on various opinions and attributes of the population (men and women) under study in more detail.

\subsection{Area of the Study and Rationale}

The study was conducted in Bundibugyo District. The district is located in the Western region of Uganda bordering the Democratic Republic of the Congo alongside several bordering districts: Ntoroko District to the northeast, Kibaale District to the east, Kabarole District to the south, and the DRC to the west and north. The district headquarters at Bundibugyo are located approximately 32 kilometres by road, west of Fort Portal, the nearest large town. This is about 72 kilometres by road, north of Kasese, the largest town in the sub-region. The geographic boundaries of the district are the Semliki River to the west, the Rwenzori mountains to the east, and Lake Albert to the north. In 2014, the national population census put the town's population at 18,823 (UBOS, 2014). Being a rugged mountainous area, physical access to the region is often difficult and is only many by foot. Bundibugyo District was selected because of its char- 
acteristics in relation to the EVD outbreak, which occurred in 2007 in Uganda.

\subsection{Study Population}

The study population consisted of males and females who are residents of communities in Bundibugyo District. These were selected purposively from areas that have ever had cases of Ebola. The study population also included residents of Bundibugyo Town Council and Kikyo Sub-County in Bundibugyo District and among the residents included men and women from local communities, health workers (medical officers, veterinary officer and village health trainees).

\subsection{Data Collection Methods}

Various methods were used to collect data or information from the study participants. The methods included Key Informant Interviews (KIIs), Focus Group Discussions (FGDs) and individual interviews using questionnaires. Gender-sensitive methods such as gender-segmented FGDs and questionnaire interviews were used so as to get perspectives of both men and women on EVD. The study also used simple ranking, one of the qualitative participatory appraisal techniques for epidemiologists, termed as Participatory Epidemiology (PE) tools [18].

\subsection{Sampling Procedure}

Purposive sampling was used to get information from key informants. Participatory Epidemiology (PE) tools like simple ranking, KIIs and FGDs were held in communities with previously affected persons.

\subsection{Sampling Size Estimation}

A sample size formula by Kish Leslie for cross-sectional studies [19] was used to estimate the sample size for the study as shown in Equation (3.1).

$$
n=\frac{Z^{2} p q}{e^{2}}
$$

where $n=$ required sample size;

$Z=$ the normal curve constant that represented the level of confidence (1.96);

$e=$ the desired level of precision estimated at $95 \%$;

$p=$ the estimated proportion of attribute present in a population (70\%) and $q$ is the $1-p$.

In this study, the estimated error margin was $5.6 \%(0.056)$. This therefore results into the confidence interval at $95 \%$. It is also estimated that $70 \%$ of the population had knowledge about EVD in Bundibugyo District.

$$
254=\frac{1.96^{2} \times 0.7 \times 0.3}{0.056^{2}}
$$

By substituting for $n=254$ individuals.

Therefore, a total of 254 individuals were interviewed in Bundibugyo District using questionnaires for the quantitative approach. The study balanced the num- 
ber of men and women in the sample size. Half (or 50\%) of the respondents were men and the other $50 \%$ women from within the sample size.

\subsection{Quantitative Study}

An individual represented each household and the selection of households was done purposively based on the list of households provided by Bundibugyo District local government. Quantitatively, information from respondents was documented using a semi-structured questionnaire. The questionnaire had questions designed to answer the following; demographic data of respondents, knowledge on EVD (cause, transmission and prevention or management), attitudes and perceptions towards EVD among respondents, treatment and management of EVD, and acceptance and treatment of EVD survivors

The questionnaire was designed in English and then translated into local languages (Luganda, Rwamba and Rukonzo) before it was used, and it was re-translated into English to allow standardisation of responses across the study area as well as ensuring correct filling of the questionnaire forms and appropriate data compilation and entry. Alongside the researcher, trained interviewers administered the questionnaire in the local language to each selected participant.

\subsection{Qualitative Study}

In most qualitative research, only a sample of a population is selected purposively for any given study [20]. This study therefore used purposive sampling where the participants were chosen according to a preselected criterion that was relevant to a particular research question. For example, three FGDs (comprising of 10 members in each group) and 6 KIIs (consisting of knowledgeable men and women in particular positions in the communities) were administered during the study. These were conducted using unstructured interview guides which were administered face-to-face with the participants. The interview guides were used to probe perceptions, attitudes, practices, beliefs, experiences depending on the persons in question.

\subsection{Eligibility Criteria}

\subsubsection{Inclusion Criteria}

Only respondents or participants from Bundibugyo District were considered for this study. These respondents included those who were living in the study areas during the EVD outbreak, Ebola survivors and families that lost their loved ones due to EVD. To this end therefore, the respondents consented to the arrangement of being interviewed.

\subsubsection{Exclusion Criteria}

Immigrant settlers who had lived less than ten years in the study areas of Bundibugyo District were excluded from being interviewed. Also persons who lived in communities in which Ebola Virus Disease was unknown or had never been experienced were excluded from the study. This is because such persons were 
assumed to be having less or no knowledge to share about EVD.

\subsection{Validity and Reliability}

Only the quantitative research tool (questionnaire) was pre-tested using some of the residents of Bundibugyo District who were not included in the study sample. After making corrections, a final print of the research tool was produced for actual fieldwork.

\subsection{Data Management and Analysis}

Data was collected continuously within three months. Responses to FGDs and Key Informant Interviews (KIIs) were collected, checked, cleaned and anomalies corrected immediately. Qualitative data was analyzed in themes as shown in the data collection tools. Themes that emerged from individual statements of the participants were summarized. The qualitative data was later used to compliment the quantitative or questionnaire data. The analyzed data were presented in tables, percentages, and figures and described. More qualitative data obtained from the KIIs and FGDs was subjected to manifest content analysis. Emerging thematic areas were identified; narratives from the transcriptions were read through, coded and followed by grouping of content. Triangulation of findings from the different methodologies was done at analysis and report writing. Raw data was obtained from the field, analyzed and coded on a daily basis. This enabled the researcher to vividly recall salient issues.

Quantitative data were entered into Statistical Package for Social Scientists (SPSS) Version 22 statistical software for analysis. Tables and figures containing different variables were drawn and the frequency for each response was worked out and percentages later compared as shown.

Cross tabulations and Pearson Chi-Square $\left(\chi^{2}\right)$ tests were also performed to establish existing relationships between the study variables at $95 \%$ confidence interval. For example, chi-square tests were used to establish gender differences in knowledge about EVD, its control, attitudes and perceptions.

\section{Results}

A number of socio-demographic characteristics and their levels of analysis were recorded. These included ages and gender of the respondents, education levels attained and marital status of the respondents.

Age and gender: The study results indicate that majority of the respondents aged between 30 and 49 years of age were males. There were more females in the 50 - 59 age group compared to males. Education: More women (55.8\%) compared to men (44.2\%) had attained secondary education.

Marital Status: In terms of marital status, more men (51.3\%) compared to women $(48.7 \%)$ were married, followed by about $(52.6 \%)$ of men and $(47.4 \%)$ of women who were still single.

Religion: About (59.2\%) of the women and (40.8\%) men were Catholics by re- 
ligion, followed by (55.1\%) of the women and about $(44.9 \%)$ of the men were Protestants. The study showed that there was no significant relationship between most of the socio-demographic characteristics of the respondents and their gender since the $\mathrm{p}$-values were greater than the critical value of 0.05 at $95 \%$ confidence interval as shown in Table 1.

Knowledge, Attitudes and Perceptions about Ebola Virus Disease (EVD): The Level of knowledge about EVD and risk factors in this study was described as follows: both men and women in Bundibugyo District were interviewed about their knowledge on EVD and its risk factors. The men and women were asked whether they had ever heard about EVD, whether they can identify a person with EVD, whether they saw themselves as being at a risk of contracting EVD and reasons, and the control measures as presented in Table 2.

Table 1. Percentage distribution of respondents by social demographic characteristics.

\begin{tabular}{|c|c|c|c|c|}
\hline \multirow{2}{*}{ Socio-demographic characteristics } & \multicolumn{2}{|c|}{ Gender } & \multirow{2}{*}{$\chi^{2}$-value } & \multirow{2}{*}{ P-value } \\
\hline & Male & Female & & \\
\hline Age of the respondent $(n=252)$ & & & 5.288 & 0.259 \\
\hline Less 20 years & $14(41.2 \%)$ & $20(58.8 \%)$ & & \\
\hline $20-29$ years & $41(46.6 \%)$ & $47(53.4 \%)$ & & \\
\hline 30 - 39 years & $41(59.4 \%)$ & $28(40.6 \%)$ & & \\
\hline 40 - 49 years & $20(55.6 \%)$ & $16(44.4 \%)$ & & \\
\hline 50 - 59 years & $11(40.7 \%)$ & $16(59.3 \%)$ & & \\
\hline Sub-county (254) & & & 1.907 & 0.167 \\
\hline Bundibugyo TC & $60(45.8 \%)$ & $71(54.2 \%)$ & & \\
\hline Kikyo & $67(54.5 \%)$ & $56(45.5 \%)$ & & \\
\hline Education level $(\mathrm{n}=253)$ & & & 3.369 & 0.424 \\
\hline Informal Education & $23(57.5 \%)$ & $17(42.5 \%)$ & & \\
\hline Primary & $41(48.2 \%)$ & $44(51.8 \%)$ & & \\
\hline Secondary & $34(44.2 \%)$ & $43(55.8 \%)$ & & \\
\hline Post-secondary & $12(63.0 \%)$ & $10(37.0 \%)$ & & \\
\hline Marital Status $(n=249)$ & & & 7.173 & 0.067 \\
\hline Married & $78(51.3 \%)$ & $74(48.7 \%)$ & & \\
\hline Single & $41(52.6 \%)$ & $37(47.4 \%)$ & & \\
\hline Widowed & $2(15.4 \%)$ & $11(84.6 \%)$ & & \\
\hline Other (divorced, cohabiting) & $2(33.3 \%)$ & $4(66.7 \%)$ & & \\
\hline Religion ( $n=246$ ) & & & 2.367 & 0.124 \\
\hline Catholic & $31(40.8 \%)$ & $45(59.2 \%)$ & & \\
\hline Protestant & $31(44.9 \%)$ & $38(55.1 \%)$ & & \\
\hline Muslim & $26(60.5 \%)$ & $17(39.5 \%)$ & & \\
\hline Born Again & $9(64.3 \%)$ & $5(35.7 \%)$ & & \\
\hline SDA & $26(60.5 \%)$ & $17(39.5 \%)$ & & \\
\hline Other & $0(0 \%)$ & $1(100 \%)$ & & \\
\hline
\end{tabular}


Table 2. Level of knowledge about Ebola Viral Disease (EVD) by gender.

\begin{tabular}{|c|c|c|c|c|}
\hline \multirow{2}{*}{ Level of knowledge about EVD } & \multicolumn{2}{|c|}{ Gender } & \multirow{2}{*}{$x^{2}$-value } & \multirow{2}{*}{ P-value } \\
\hline & Male & Female & & \\
\hline Have you heard about EVD $(\mathrm{n}=254)$ & & & 2.065 & 0.141 \\
\hline Yes & $125(50.8 \%)$ & $121(49.2 \%)$ & & \\
\hline No & $2(2.5 \%)$ & $6(75.0 \%)$ & & \\
\hline \multicolumn{5}{|l|}{ Identify a person with EVD $(n=236)$} \\
\hline Yes & $116(51.8 \%)$ & $108(48.2 \%)$ & 1.552 & 0.213 \\
\hline No & $4(33.3 \%)$ & $8(66.7 \%)$ & & \\
\hline At risk of contracting $\operatorname{EVD}(\mathrm{n}=235)$ & & & 0.676 & 0.411 \\
\hline Yes & $104(51.7 \%)$ & $97(48.3 \%)$ & & \\
\hline No & $15(44.1 \%)$ & $19(55.9 \%)$ & & \\
\hline Reasons of being at risk $(n=138)$ & & & 9.095 & 0.168 \\
\hline Close contact with infected person & $22(56.4 \%)$ & $17(43.6 \%)$ & & \\
\hline Dangerous disease & $15(57.7 \%)$ & $11(42.3 \%)$ & & \\
\hline EVD survivors still alive & $6(60.0 \%)$ & $4(40.0 \%)$ & & \\
\hline EVD does not show signs & $16(50.0 \%)$ & $16(50.0 \%)$ & & \\
\hline Wild animals still exist & 0 & $1(100.0 \%)$ & & \\
\hline Health workers are at risk & $2(28.6 \%)$ & $5(71.4 \%)$ & & \\
\hline Being in area affected by Ebola & $6(26.1 \%)$ & $17(73.9 \%)$ & & \\
\hline Know control of EVD $(n=245)$ & & & 0.534 & 0.465 \\
\hline Yes & $120(50.6 \%)$ & $117(49.4 \%)$ & & \\
\hline No & $3(37.5 \%)$ & $5(62.5 \%)$ & & \\
\hline Control measures of EVD outbreak $(n=247)$ & & & 0.685 & 0.710 \\
\hline Avoiding contact with blood/body fluids & $71(51.1 \%)$ & $68(48.9 \%)$ & & \\
\hline Avoiding funeral rituals & $25(45.5 \%)$ & $30(54.5 \%)$ & & \\
\hline Suspected patient goes to hospital & $28(52.8 \%)$ & $25(47.2 \%)$ & & \\
\hline Protection from EVD $(n=251)$ & & & 2.564 & 0.464 \\
\hline Vaccination & $12(66.7 \%)$ & $6(33.3 \%)$ & & \\
\hline Avoid contact with sick wild animals & $27(51.9 \%)$ & $25(48.1 \%)$ & & \\
\hline Traditional medicine & $8(50.0 \%)$ & $8(50.0 \%)$ & & \\
\hline Avoid contact with EVD infected person & $78(47.3 \%)$ & $87(52.7 \%)$ & & \\
\hline
\end{tabular}

The level of knowledge about EVD and its risk factors (whether men and women had heard about EVD, whether they can identify a person with EVD, their perception of being at a risk of contracting EVD, control and protection measures) by gender was not statistically significant. Most of the P-values were greater than the critical value of 0.05 at $95 \%$ confidence interval.

The study findings show that slightly more males (50.8\%) than females (49.2\%) 
had heard about Ebola Virus Disease (EVD) in Bundibugyo District. Among those who had heard about EVD, more males (51.8\%) than females (48.2\%) were able to identify a person with the disease. Figure 1 shows that the majority of males indicated vomiting and high fever as the major symptoms of EVD while most females said that diarrhoea and bleeding were the major symptoms of EVD in Bundibugyo District. Qualitative study also revealed that the mixed FGD participants also described the severity of EVD and its symptoms as follows:

"It is a serious disease, it kills in a short time, It is a serious disease and it kills in seven days or two weeks, It is a transmitted disease, It came unknowingly that it is a serious disease and it killed people, it is easily transmitted with a person who is sick, you get the disease immediately, When someone gets sick, he could get isolated. The signs and symptoms they mentioned include: high fever, bleeding from sores, ears, nose, eyes, the eyes are itchy as if they have poured sand in your eyes, finally, the EVD patient gets joint pains, high fever, general body weakness, vomiting, diarrhoea, loss of appetite, coughing" (FGD with both Males and Females, Bundibugyo Town Council).

Furthermore, one of the Key Informants added:

"Ebola is a haemorrhagic fever that affects a person. The disease has signs and symptoms that are easily seen like the running nose, bleeding from everywhere in faeces, nose, even the skin, headache, malaise" (FemaleNursing Officer, Ntandi Health Centre III, Kikyo Sub County).

Community members were also asked whether they were at risk of contracting EVD and more males (51.7\%) than females (48.3\%) admitted being at risk. The most common reasons of being at risk of EVD mentioned by the respondents included: close contact with infected persons, being in an area (or surrounded by an area) affected by EVD as well as the fact that EVD may not easily show signs which makes it easy to spread from one person to another. Similar reasons were reported in the FGD with men:

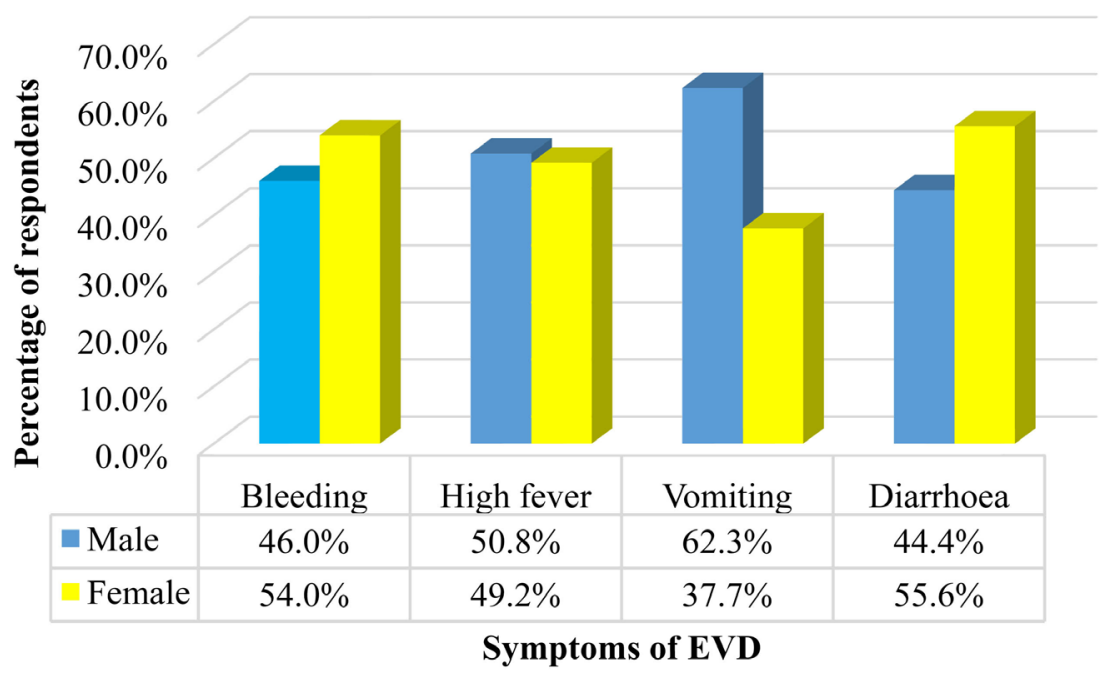

Figure 1. Showing symptoms of Ebola Virus Disease by gender. 
"Firstly, we think we are at risk because Ebola is common with the bordering places in other countries, like Bundibugyo is bordering Congo, then if you go to Gulu, its bordering Sudan, so that is why there is a high chance of getting Ebola. Secondly, it is due to movements, we do move a lot, we go to Kampala, where, everywhere, getting it is easy so it could come in transport. Thirdly, it is believed to come from animals and people are still hunting, they have not stopped poaching. People still feed on bush meat and they say it is very delicious. Fourthly, even like gatherings like we are here, if one is a victim then you find that all of us have acquired it. Lastly, members are at risk because we are still shaking hands and if someone who is infected is here, what will happen? So you never know" (FGD with Men, Kikyo H.C.IV, Kikyo Sub-County).

Also the senior nursing officer accepted that the medical team rushed at the scene of the suspected Ebola case and this itself puts them at risk more especially in absence of protective materials;

"Before they knew it was Ebola, they selected some health workers and got a site where to treat that disease that had come in and when they came to know that it was really Ebola, they requested for the government to come in and give assistance, and health lives of medical officers at site were at risk' (Senior Nursing Officer-Bundibugyo Hospital).

According to the control measures of EVD, It was revealed that equal numbers of the respondents also said that they knew about the control of EVD outbreak. The most control measure of EVD during outbreaks mentioned was avoiding of contact with blood or body fluids as well as avoiding of funeral rituals including touching of dead bodies. In this case, more males (51.1\%) than females (48.9\%) mentioned avoiding of contact with blood or body fluids. More females $(54.5 \%)$ than males proposed avoiding of funeral rituals. More males (52.8\%) than females (47.2\%) said that the suspected EVD patients should be taken to the hospital immediately. However these differences were not statistically significant at 0.05 level of significance (as shown in Table 2).

When asked on how community members could be protected from EVD, more females (52.7\%) than males (47.3\%) proposed avoiding contact with EVD infected persons. More males (51.9\%) than females proposed avoiding contact with sick wild animals. Other ways of protection mentioned included vaccination and traditional medicine among others. The community members were also asked on the best ways of eliminating Ebola Virus Disease during an outbreak and the results disaggregated by gender are presented in Table 3.

As a method of eliminating EVD from Bundibugyo District, more males (54.7\%) than females (45.3\%) proposed avoiding body contact through for instance shaking hands and kissing. Similarly, more males (61.1\%) than females (38.9\%) proposed sensitization of masses on EVD as the best way of eradicating it during an outbreak. On the other hand, more females (72.0\%) than males (28.0\%) proposed burning of materials used by EVD patients. Also more females (60.0\%) than males (40.0\%) said that protective gear should be used while handling EVD patients. It is indicated that only half (50.0\%) and females also only 
Table 3. Ways of eradicating EVD during an outbreak by gender.

\begin{tabular}{cccc}
\hline \multirow{2}{*}{ Ways of eradicating EVD during an outbreak } & \multicolumn{2}{c}{ Gender } & \multirow{2}{*}{ Total } \\
\cline { 2 - 3 } & Male & Female & \\
\hline Avoiding body contact e.g. shaking hands & $29(54.7 \%)$ & $24(45.3 \%)$ & $53(100.0 \%)$ \\
Sensitize masses & $22(61.1 \%)$ & $14(38.9 \%)$ & $36(100.0 \%)$ \\
Isolate Ebola victims (isolation camps) & $2(22.2 \%)$ & $7(77.8 \%)$ & $9(100.0 \%)$ \\
Employ more health care workers & $2(40.0 \%)$ & $3(60.0 \%)$ & $5(100.0 \%)$ \\
Treating Ebola infected people immediately & $7(63.6 \%)$ & $4(36.4 \%)$ & $11(100.0 \%)$ \\
Discourage eating bush meat & $7(50.0 \%)$ & $7(50.0 \%)$ & $14(100.0 \%)$ \\
Test blood samples of Ebola disease suspects & 0 & $1(100.0 \%)$ & $1(100.0 \%)$ \\
Avoid burial rituals on Ebola dead corpses & $2(33.3 \%)$ & $4(66.7 \%)$ & $6(100.0 \%)$ \\
Burn materials used by EVD patients & $7(28.0 \%)$ & $18(72.0 \%)$ & $25(100.0 \%)$ \\
Washing hands and utensils using detergents e.g. jik & $2(40.0 \%)$ & $3(60.0 \%)$ & $5(100.0 \%)$ \\
Use protective gear while handling Ebola patients & $6(40.0 \%)$ & $9(60.0 \%)$ & $15(100.0 \%)$ \\
Total & $86(47.8 \%)$ & $94(52.2 \%)$ & $180(100.0 \%)$ \\
& $\chi^{2}=13.022, \mathrm{P}$-value $=0.222$ \\
\hline
\end{tabular}

(50.0\%) said that eating of bush meat should be discouraged as a form of eliminating EVD. Other ways of eliminating EVD as proposed by more females included: creation of EVD isolation centres, employment of more health workers, testing of blood samples for EVD suspects, avoiding of burial rituals as well as washing hands and utensils using detergents like jik and soap. More males (63.6\%) than females (36.4\%) proposed that Ebola Virus Disease infected persons should be treated immediately as a mechanism of eliminating EVD from the community. Some female FGD participants mentioned similar and other ways of eliminating the outbreak of EVD:

"Normally the VHTs in our community sensitize the people about sanitation and hygiene and if Ebola could erupt because of sanitation and hygiene, they would know since they are more aware about hygiene and sanitation, Avoid contacting each other during the outbreak, avoiding to eat dead animals, any dead animal, avoiding crowded places, avoid touching someone who has died and you are not aware of what has killed him, avoid shaking hands while greeting, avoid sharing dresses, and use of protective gears when handling Ebola patients" (FGDs with women, Kikyo H. C. IV, Kikyo Sub-County).

A village health team member said that measures were put in place to contain the spread of EVD during the outbreak in Bundibugyo. He added that a health team formed a group to bury the bodies while the community greatly participated in digging graves:

"Okay the community apart from sensitization and mobilization, the burial system was not for the community because when someone died the only thing was to go and dig the pit but the burial ceremonies were for the health workers. 
Because there was a team, the burial team was from the health unit" (Male V. H. T, Kikyo H. C. IV, Kikyo Sub-County).

According to the attitudes towards Ebola Viral Disease Survivors, the respondents were asked about their attitudes towards EVD survivors and the results are presented in Table 4. It indicated that more males $(51.2 \%)$ than females (48.8\%) said they could relate with EVD survivors. This is a sign of positive attitudes toward former patients of EVD. Among the few who said that they could not relate with EVD survivors, the majority were females (52.2\%). The reasons given here by the majority of females $(58.5 \%)$ were that they feared contracting the disease while most males (56.4\%) they feared stigma from the community. Similarly, a big proportion of the community members were willing to buy from a shop of an EVD survivor. For instance, as shown in Table 4 an equal proportion of both males and females said that they would buy goods from a shop of an EVD survivor. This indicates that the majority of the community members had positive attitudes towards Ebola Virus Disease survivors in Bundibugyo District. FGDs with female participants in Bundibugyo Town Council also revealed that the community had developed a good attitude towards EVD survivors especially after the declaration of Bundibugyo District as Ebola free:

"When Bundibugyo District was declared Ebola free, we made a party, we were free with them. We started relating with the survivors but during the outbreak, we discriminated them since we feared getting sick" (FGD with Females, Bundibugyo Town Council).

However, one of the Key Informants revealed that the community had reservations in dealing with Ebola Virus Disease patients until Bundibugyo was declared EVD free:

Table 4. Attitudes towards EVD survivors by gender.

\begin{tabular}{|c|c|c|c|c|}
\hline \multirow{2}{*}{ Differences in attitudes } & \multicolumn{2}{|c|}{ Gender } & \multirow{2}{*}{$x^{2}$-value } & \multirow{2}{*}{ P-value } \\
\hline & Male & Female & & \\
\hline Can relate with EVD survivor $(\mathrm{n}=254)$ & & & 0.273 & 0.602 \\
\hline Yes & $83(51.2 \%)$ & $79(48.8 \%)$ & & \\
\hline No & $44(47.8 \%)$ & $48(52.2 \%)$ & & \\
\hline Reasons for not relating with EVD survivors $(n=92)$ & & & 1.999 & 0.157 \\
\hline Fear of contracting disease & $22(41.5 \%)$ & $31(58.5 \%)$ & & \\
\hline Fear of stigma from community & $22(56.4 \%)$ & $17(43.6 \%)$ & & \\
\hline Buy from the shop of an EVD survivor $(n=251)$ & & & 0.014 & 0.907 \\
\hline Yes & $97(50.0 \%)$ & $97(50.0 \%)$ & & \\
\hline No & $28(49.1 \%)$ & $29(50.9 \%)$ & & \\
\hline Keep information secret if family member contracts Ebola $(n=225)$ & & & 0.015 & 0.902 \\
\hline Yes & $26(51.0 \%)$ & $25(49.0 \%)$ & & \\
\hline No & $87(50.0 \%)$ & $87(50.0 \%)$ & & \\
\hline
\end{tabular}


"First of all, there was isolation of Ebola patients and when this happened, communication was lost, of meeting together, staying together. Not until we were told that Ebola has completely gone, things went back to normal' (Female Senior Nursing Officer, Bundibugyo Hospital).

On another positive note, most of the community members $(50.0 \%$ of males and $50.0 \%$ of females) were determined not to keep information secret in case a family member contracted EVD. There were however, a significant number of respondents that said that they would keep information secret if a family member contracted EVD (51.0\% of males and $50.0 \%$ of females out of 51 respondents that said yes). Keeping secrets on EVD is quite dangerous in a community because it increases the risk of disease spread as well as the associated human losses.

According to the Perceptions about the Causes, Transmission and Remedy of EVD, Respondent's perceptions about the causes, transmission and treatment of EVD are presented in Table 5. The study indicated that whereas the study respondents had different opinions about the causes, modes of transmission as

Table 5. Perceptions of causes, transmission and remedy for EVD by gender.

\begin{tabular}{|c|c|c|c|c|}
\hline \multirow{2}{*}{ Perceptions towards Ebola Virus Disease } & \multicolumn{2}{|c|}{ Gender } & \multirow{2}{*}{$\chi^{2}$-value } & \multirow{2}{*}{ P-value } \\
\hline & Male & Female & & \\
\hline Ebola exists $(n=241)$ & & & 0.092 & 0.762 \\
\hline Yes & $109(49.8 \%)$ & $110(50.2 \%)$ & & \\
\hline No & $9(40.9 \%)$ & $13(59.1 \%)$ & & \\
\hline Cause of EVD outbreaks $(n=254)$ & & & 2.581 & 0.764 \\
\hline Virus & $61(47.7 \%)$ & $67(52.3 \%)$ & & \\
\hline Bats/monkey/other wild animals & $63(52.5 \%)$ & $57(47.5 \%)$ & & \\
\hline God/any other supreme being & $1(50.0 \%)$ & $1(50.0 \%)$ & & \\
\hline Witchcraft & $1(50.0 \%)$ & $1(50.0 \%)$ & & \\
\hline Sinning & 0 & $1(100.0 \%)$ & & \\
\hline Curse & $1(100.0 \%)$ & 0 & & \\
\hline EVD transmission mode $(n=249)$ & & & 5.738 & 0.453 \\
\hline Body contact & $64(48.1 \%)$ & $69(51.9 \%)$ & & \\
\hline Through air & $4(80.0 \%)$ & $2(20.0 \%)$ & & \\
\hline Needle pricks & $9(69.2 \%)$ & $4(30.8 \%)$ & & \\
\hline Contact with animals & $9(45.0 \%)$ & $11(55.0 \%)$ & & \\
\hline Contact with bats & $3(37.5 \%)$ & $5(62.5 \%)$ & & \\
\hline Contact with body fluids & $36(52.2 \%)$ & $33(47.8 \%)$ & & \\
\hline Insect bites & $0(0 \%)$ & $1(100 \%)$ & & \\
\hline Remedy for Ebola Virus Disease $(n=250)$ & & & 3.453 & 0.327 \\
\hline Traditional medicine & $3(33.3 \%)$ & $6(66.7 \%)$ & & \\
\hline Spiritual healing & $5(31.3 \%)$ & $11(68.8 \%)$ & & \\
\hline Modern medicine & $105(50.7 \%)$ & $102(49.3 \%)$ & & \\
\hline Herbal medicine & $10(55.6 \%)$ & $8(44.4 \%)$ & & \\
\hline
\end{tabular}


well as the remedies for EVD in Bundibugyo District. Their perceptions were not statistically significant by gender. More females (50.2\%) than males (49.8\%) acknowledged that EVD exists. On the other hand, there a good number of respondents reported that EVD did not exist (22 out of 241 respondents). Among those that denied existence of EVD, the majority were females (59.1\%).

When asked about the causes of EVD, more females (52.3\%) than males (47.7\%) attributed it to a virus. This was followed by respondents who indicated that animals were the cause of Ebola Virus Disease (males were $52.5 \%$ and females were 47.5\%). Other respondents said that the cause of Ebola Virus Disease was a curse from God and associated with witchcraft and sinning.

The respondents who indicated that the cause of Ebola Virus Disease was attributed to animals were further asked how animals cause EVD and the findings are shown in Figure 2 showed that an equal number of males (50.0\%) and females $(50.0 \%)$ said that EVD is transmitted through animals. More females (66.7\%) than males (33.3\%) said that EVD is transmitted through pigs. Similarly, more females than females also said that EVD is transmitted through poultry. More males (50.9\%) than females (49.1\%) said that monkeys were responsible for the transmission of Ebola Virus Disease. Equal proportions of males and females attributed the transmission of EVD to bats and antelopes. More females (75.0\%) than males (25.0\%) said that Ebola Virus Disease is transmitted through wild pigs.

On human-to-human transmission of EVD, more females (51.9\%) than males (48.1\%) reported body contact between an infected person and a non-infected person. More males $(52.2 \%)$ than females $(47.8 \%)$ said that contact with body fluids is also responsible for the transmission of EVD. More females (55.0\%) than males (45.0\%) said that contact with animals was responsible for the transmission of EVD. However, the rest of the respondents also cited several ways through which EVD is transmitted and these included: through air, through needle pricks, contact with bats as well as insect bites.

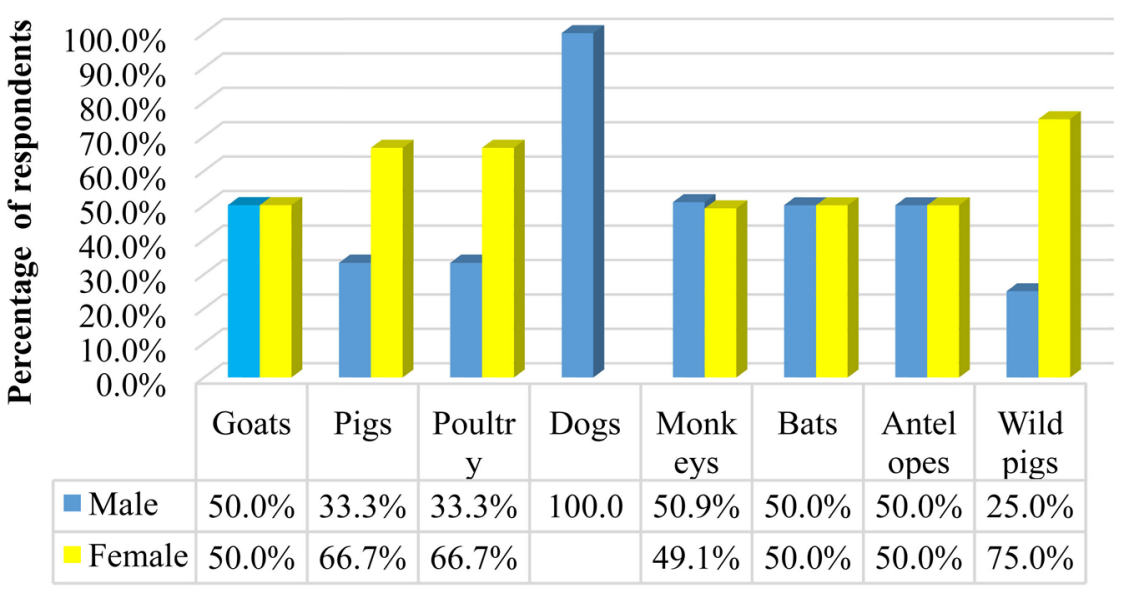

\section{EVD transmission through Animals}

Figure 2. Community perceptions of animals that transmit ebola virus disease. 
Similarly, some male FGD participants had different right and wrong ideas on what EVD was and how it was transmitted, citing similarities with other diseases such as cholera and AIDS:

"Ebola must be Cholera. It is associated with cannibalism/ spirits, or caused by eating of dead apes like monkeys and gorillas, poor hygiene. Ebola must be another type of AIDS because some people in the community play sex with animals and some signs of AIDS resemble for this disease like diarrhoea. However, there was a sudden death of a white tourist during that time of the disease onset, so the community thought he brought the disease" (FGDs with Males, Kikyo H.C.IV).

Nonetheless, most of the medical key informant knew the right causes of EVD. For example:

"Well, there are very many causes that we believe Ebola come from. We believe it could come from bats, monkeys, people who eat wild meat, most of the research shows that it comes from those things" (Female Medical Records Officer, Bundibugyo District).

The study respondents in Bundibugyo District were also asked ways through which the Ebola Virus Disease can be healed (See Table 4.2.3). The results show that more males (50.7\%) than females (49.3\%) said that modern medicine is the best way of healing Ebola Virus Disease. Similarly, more males (55.6\%) than females (44.4\%) said that herbal medicine was another way of healing Ebola Virus Disease. Other modes of healing EVD mentioned included traditional medicine and spiritual healing.

\section{Discussion}

With regard to the socio-demographic characteristics of the participants, an equal number of both males $(n=127)$ and females $(n=127)$ was selected and this was meant to seek for views from all people on EVD. Women take the leading role in caring for their families. For instance nurses are predominantly female and are at greater risk of getting sick during EVD outbreaks (The Lancet, 2014). Men on the other hand men play a significant role of transporting the suspected EVD patients to hospitals. According to the findings, more females than males had attended secondary education. However, the figures for men and women from the study are lower at only $44.2 \%$ and $55.8 \%$ respectively compared with the national literacy rate of $84.7 \%$. Uganda's Ministry of Education and Sports has also recorded a higher percentage $(52 \%)$ of males who enrolled secondary education compared to the females whose ratios reduce as they go higher in their education [21]. Gender differences in religious beliefs were considered as important. Some religious beliefs still exist in the society like some religions believed that prayers alone would eliminate Ebola from their villages. The Muslims' practice of washing dead bodies before burial is a huge risk factor in terms of increasing the spread of EVD. In the same instance, Christians especially Catholics and protestants spend some days before burying the dead in 
Bundibugyo, the burial team, dominated by men was often hired and paid some money during the EVD outbreak. This is in contrast to what happens among the Acholi in Northern Uganda, where the older women are expected to prepare bodies for burial, putting them at a higher risk compared to men [11]. However, there was no significant relationship between most of the socio-demographic characteristics of the respondents and their gender since the p-values were greater than the critical value of 0.05 at $95 \%$ confidence interval. Community efforts to block traditional burials that could increase the risk of men and women have been reported in other parts of Africa, such as Nigeria where communities blocked the traditional burials of the ashes of a nurse who had died from Ebola [22].

With regard to knowledge and awareness of EVD, this study found out that more females than males believed that EVD exists. Again, more females than males rightly said that EVD is caused by a virus. Whereas an equal number of males and females said EVD is caused by bats, goats and antelopes, a number of males associated it with wild animals, pigs, and poultry. Studies that have disaggregated these causes of EVD by gender are difficult to come by, but the men and women of Bundibugyo were right on bats as they have previously been identified as vectors of the virus [9].

In terms of practices and community's experiences in regards to the causes, signs, transmission mechanisms, treatment and control measures, these findings are in line with the general knowledge that Ebola is caused by a virus. However, only a proportion (50.4\%) of the respondents believed that EVD is caused by a virus. Only $47.2 \%$ of the respondents associated eating of wild animals like monkeys and bats to Ebola transmission. This is much lower than expected since people in this area often hunt game reserve. In addition monkeys in the area are often hunted for food [11]. These findings therefore are likely to have been influenced by fear as hunting game for food in the nearby Semuliki Game reserve was illegal.

This study also observed that only a small proportion of men and women believe EVD is caused by a curse from God or witchcraft (0.8\%) In this study, most of the respondents (97.9\%) - men and women knew that among the various ways of controlling the transmission of EVD in the community is avoiding burial rituals or washing dead bodies and delayed burials of one or two days. This finding is similar to what [17] found to be associated with traditional practices in northern Uganda. Traditionally among the Acholi women are responsible for cleansing the dead before burial. This study showed that men, compared to women were considered to be at great risk of contracting the EVD. This is possibly because they travel a lot, commonly buried the dead and took Ebola patients to hospitals among other tasks. It was established [17] that men are more likely to have contact with forest animals including handling and butchering of animals found dead in forests.

Community's Experiences about EVD and Its Effects

In this study, most of the respondents (73.5\%) argued that EVD mostly af- 
fected men compared to the women (26.5\%). Previous studies have not clearly indicated this gender difference in effects of EVD. Studies in other parts of Africa (mainly Liberia and Sierra Leone) have also shown that women's caregiving role exposes them more to the virus and therefore increases their fatalities during EVD outbreaks [23] [24]. The study showed that more female respondents than men perceived that EVD outbreaks impacted the education system. They mentioned most schools getting closed and sending home sick students/pupils and banning the sharing of scholastic materials. A study by [25] also reported that community members stopped their children from attending school in Gulu and Luweero Districts in Uganda following the EVD outbreak. This was due to the general fear of contracting the disease. The observed findings showed that EVD affected the development of the community and increased women and men's vulnerability to adverse socio economic outcomes. Most people and investors were not maximally working and put their operations on hold which resulted into decline of business activities.

\section{Conclusion}

It is evident that women and men know the right and also the wrong causes of EVD, however, most women have less knowledge on causes and transmission. There were several misconceptions on the disease dynamics as some respondents even reported that EVD could be treated using traditional medicine. Concerted efforts to make community members especially females aware of the right causes, transmission and prevention techniques of EVD are needed at community level. Some bad cultural practices such as hunting and eating bush meat mostly practiced by men were suspected although not admitted by most of the respondents. Therefore, there is a need to sensitise communities in order to improve negative attitudes and perceptions and reduce discrimination and stigma.

\section{Conflicts of Interest}

The authors declare no conflicts of interest regarding the publication of this paper.

\section{References}

[1] CDC (2014) 2014 Ebola Outbreak in West Africa. Centers for Disease Control and Prevention, 1 .

[2] Towner, J.S., Sealy, T.K., Khristova, M.L., Albariño, C.G., Conlan, S., Reeder, S.A. and Nichol, S.T. (2008) Newly Discovered Ebola Virus Associated with Hemorrhagic Fever Outbreak in Uganda. PLoS Pathogens, 4, e1000212. https://doi.org/10.1371/journal.ppat.1000212

[3] CDC and WHO (1998) Infection Control for Viral Haemorrhagic Fevers in the African Health Care Setting. Centers for Disease Control and Prevention: National Center for Infectious Diseases, Division of Viral and Rickettsial Diseases, Special Pathogens Branch, WHO Collaborating Centre for Viral Haemorrhagic Fevers \& World Health Organization: Division of Emer, 198.

[4] MacNeil, A., Farnon, E.C., Wamala, J., Okware, S., Cannon, D.L., Reed, Z. and 
Rollin, P.E. (2010) Proportion of Deaths and Clinical Features in Bundibugyo Ebola Virus Infection, Uganda. Emerging Infectious Diseases, 16, 1969-1972. https://doi.org/10.3201/eid1612.100627

[5] WHO (2014) Ebola Virus Disease in West Africa-The First 9 Months of the Epidemic and Forward Projections. The England New Journal of Medicine, 371, 1481-1495. https://doi.org/10.1056/NEJMoa1411100

[6] Raykar, M.H., Shinde, R.V. and Shyale, S.S. (2014) Ebola Virus Disease. Pharmtechmedica (PhTechMed), 3, 493-496.

[7] Abramowitz, S.A., McLean, K.E., McKune, S.L., Bardosh, K.L., Fallah, M., Monger, J. and Omidian, P.A. (2015) Community-Centered Responses to Ebola in Urban Liberia: The View from Below. PLoS Neglected Tropical Diseases, 9, e0003706. https://doi.org/10.1371/journal.pntd.0003706

[8] World Health Organization (2015) WHO | Ebola Vaccines, Therapies, and Diagnostics.

[9] Leroy, E.M., Kumulungui, B., Pourrut, X., Rouquet, P., Hassanin, A., Yaba, P. and Swanepoel, R. (2005) Fruit Bats as Reservoirs of Ebola Virus. Nature, 438, 575-576. https://doi.org/10.1038/438575a

[10] Shoemaker, T., MacNeil, A., Balinandi, S., Campbell, S., Wamala, J.F., McMullan, L.K. and Nichol, S.T. (2012) Reemerging Sudan Ebola Virus Disease in Uganda, 2011. Emerging Infectious Diseases, 18, 1480-1483. https://doi.org/10.3201/eid1809.111536

[11] Okware, S.I., Omaswa, F.G., Zaramba, S., Opio, A., Lutwama, J.J., Kamugisha, J., Lamunu, M., et al. (2001) An Outbreak of Ebola in Uganda. Tropical Medicine and International Health, 7, 1068-1075. https://doi.org/10.1046/j.1365-3156.2002.00944.x

[12] Chertow, D.S., Kleine, C., Edwards, J.K., Scaini, R., Giuliani, R. and Sprecher, A. (2014) Ebola Virus Disease in West Africa-Clinical Manifestations and Management. The New England Journal of Medicine, 2057-2059.

[13] Kobayashi, M., Beer, K.D., Bjork, A., Chatham-Stephens, K., Cherry, C.C., Arzoaquoi, S., Nyenswah, T.G., et al. (2015) Community Knowledge, Attitudes, and Practices Regarding Ebola Virus Disease-Five Counties, Liberia, September-October, 2014. MMWR Morbidity and Mortality Weekly Report, 64, 714-718.

[14] Trad, M., Fisher, D.A. and Tambyah, P.A. (2014) Ebola in West Africa. The Lancet Infectious Diseases, 14, 1045. https://doi.org/10.1016/S1473-3099(14)70924-7

[15] Roddy, P., Howard, N., van Kerkhove, M.D., Lutwama, J., Wamala, J., Yoti, Z., Borchert, M., et al. (2012) Clinical Manifestations and Case Management of Ebola Haemorrhagic Fever Caused by a Newly Identified Virus Strain, Bundibugyo, Uganda, 2007-2008. PLoS ONE, 7, 2007-2008.

https://doi.org/10.1371/journal.pone.0052986

[16] Kinsman, J. (2012) “A Time of Fear": Local, National, and International Responses to a Large Ebola Outbreak in Uganda. Globalization and Health, 8, 15. https://doi.org/10.1186/1744-8603-8-15

[17] Hewlett, B.S. and Amolat, R.P. (2003) Cultural Contexts of Ebola in Northern Uganda. Emerging Infectious Diseases, 9, 1242-1248.

https://doi.org/10.3201/eid0910.020493

[18] Swai, E.S. and Neselle, M.O. (2010) Using Participatory Epidemiology Tools to Investigate Contagious Caprine Pleuropneumonia (CCPP) in Maasai Flocks, Northern Tanzania. International Journal of Animal and Veterinary Advances, 2, 141-147.

[19] Kish, L. (1965) Survey Sampling. In: Johnson, N.L. and Smith Jr., H., Eds., System- 
atic Biology, John Wiley \& Sons, Inc., New York, Vol. 46, 643.

[20] Golafshani, N. (2003) Understanding Reliability and Validity in Qualitative Research. The Qualitative Report, 8, 597-607.

[21] UBOS (2014) 2014 Census Population. Uganda National Population and Housing Census Result.

[22] Grigg, C., Waziri, N., Olayinka, A. and Vertefeuille, J. (2015) Use of Group Quarantine in Ebola Control-Nigeria, 2014. Morbidity and Mortality Weekly Report, 64, 124.

[23] Shears, P. and O’Dempsey, T.J.D. (2015) Ebola Virus Disease in Africa: Epidemiology and Nosocomial Transmission. Journal of Hospital Infection, 90, 1-9. https://doi.org/10.1016/j.jhin.2015.01.002

[24] Chippaux, J.-P. (2014) Outbreaks of Ebola Virus Disease in Africa: The Beginnings of a Tragic Saga. Journal of Venomous Animals and Toxins Including Tropical Diseases, 20, 44. https://doi.org/10.1186/1678-9199-20-44

[25] Mbonye, A., Wamala, J., Kaboyo, W., Tugumizemo, V., Aceng, J. and Makumbi, I. (2012) Repeated Outbreaks of Viral Hemorrhagic Fevers in Uganda. African Health Sciences, 12, 579-583. 